\title{
One-dimensional Eikonal Non-Local (ENL) damage models: Influence of the integration rule for computing interaction distances and indirect loading control on damage localization
}

\author{
Flavien Thierry ${ }^{\mathrm{a}}$, Giuseppe Rastiello ${ }^{\mathrm{b}, *}$, Cédric Giry ${ }^{\mathrm{a}}$, Fabrice Gatuingt ${ }^{\mathrm{a}}$ \\ ${ }^{a}$ Université Paris-Saclay, ENS Paris-Saclay, CNRS, LMT - Laboratoire de Mécanique et Technologie, 91190, Gif-sur-Yvette, France \\ ${ }^{b}$ Université Paris-Saclay, CEA, Service d'études mécaniques et thermiques, 91191, Gif-sur-Yvette, France
}

\begin{abstract}
The Eikonal Non-Local approach models damage dependent non-local interactions by considering that interaction distances between material points are computed from solving an eikonal equation with an isotropic/anisotropic damage dependent metric Riemann function. In the finite element context, such a formulation exhibits good regularization properties and naturally allows one to model strain localization when non-local interactions vanish. In an isotropic damage mechanics framework, the damage variable tends to unity on a single integration point, and no damage diffusion occurs. In a one-dimensional context, evaluating the interaction distance between two points comes into computing an integral where the integrated function depends on the damage field between them. Desmorat et al. [9] performed the integration using a trapezoidal rule (under the assumption that the damage field is constant between adjacent integration points). Conversely, Jirásek and Desmorat [19] assumed that the damage field is linear between nearby integration points. Mainly motivated by improved integration accuracy, this method leads to a more gradual damaging process and makes it easier to follow the structural response when damage localizes. In this contribution, we show that despite these advantages, such a choice induces parasite damage diffusion issues, making the use of a standard trapezoidal rule more relevant. Moreover, once this choice is made, the structural response during damage localization can be conveniently described using a path-following method based on controlling the non-local strain variation of the localizing element instead of the local strain as in the works cited above.
\end{abstract}

Keywords: Eikonal Non-Local (ENL) damage model, Damage dependent interactions, Integration formulas, Path-following constraint equations, Regularization

\section{DOI: https://doi.org/10.1016/j. mechrescom.2020.103620}

\section{Introduction}

Continuum damage models are often used to represent failure processes in different kinds of materials. From a mathematical viewpoint, it is well known that material softening leads to the ill-posedness of the equilibrium problem. In a numerical framework, this translates into spurious energy dissipation and non-objective results with respect to the spatial discretization of the considered problem.

Several methods were proposed in the literature as localization limiters. One can cite, among them, Integral Non-Local (INL) formulations on the internal variables of the constitutive model [31, 12, 4, 15], gradient enhanced models [11, 29], phase-field formulations [10, 5, 25, 37], or Thick Level Set models [27,

\footnotetext{
*Corresponding author

Email address: giuseppe.rastiello@cea.fr(Giuseppe Rastiello)
}

3, 26, 23]. Despite their differences, the common feature of these theories is that a characteristic length is introduced into the formulation allowing to diffuse dissipation over a zone of finite size. From a numerical viewpoint, this enables recovering mesh insensitivity.

In particular, INL models $[31,2]$ are widely used due to their simplicity of implementation and numerical robustness. According to these formulations, damage evolution is driven by a non-local field (e.g., a non-local equivalent strain field), obtained by weighted averaging of the corresponding local field over the domain. In standard theories, averaging is performed such that non-local interactions between material points are controlled by the Euclidean distance between them (i.e., the higher the distance, the lower the interaction).

Limitations and deficiencies of INL formulations have been pointed out by many authors (e.g., [13, 17, 36, 1, 14, 16]). In particular, considering fixed non-local interaction distances leads to non-physical interactions through holes, cracks, and developing damaged bands. This results in parasite damage spreading across damaged bands, near notches, and free edges in simulations.

Enhancements of the original methods were proposed to face 
F. Thierry, G. Rastiello, C. Giry, F. Gatuingt / Mech. Res. Comm. / One-dimensional Eikonal Non-Local (ENL) damage models: Influence of the integration rule for computing interaction distances and indirect loading control on damage localization

these limitations (e.g., [28, 14, 20, 30]) and to describe more precisely strain localization processes in softening media through considering evolving non-local interactions.

In the Eikonal Non-Local (ENL) formulation [9], interaction distances between material points are computed by solving an isotropic time-independent Eikonal equation with a damage dependent metric function. In two- and three-dimensional (2D and 3D) settings, the ENL formulation considers that damage induces a curvature of the Riemannian space in which interaction distances are computed. This mathematical/physical framework allows for directly modeling evolving interactions during the localization process, thus naturally leading to progressive damage - to - fracture transition. In [9, 19] (uni-dimensional case, 1D) and [33] (two-dimensional case, 2D) it was shown that an isotropic ENL damage formulation allows for representing the main phases of strain localization process in quasibrittle materials, including the progressive transition from diffuse to localized damage. Furthermore, the regularization properties of standard INL formulations are preserved until high damage levels.

In the $1 \mathrm{D}$ setting that is considered in this work, finding the interaction distance $\tilde{\ell}_{x x^{\prime}}=\tilde{\ell}_{x}\left(x^{\prime}\right)$ between two material points $x$ and $x^{\prime}$ belonging to a domain $\Omega$ consists in solving the eikonal equation:

$$
\begin{array}{r}
\sqrt{1-D(\eta)}\left|\frac{\mathrm{d}}{\mathrm{d} \eta} \tilde{\ell}_{x}(\eta)\right|=1 \\
\tilde{\ell}_{x}(\eta=x)=0
\end{array}
$$

with $D(\eta) \in[0,1)$ the isotropic scalar-valued damage field. After integrating between $x$ and $x^{\prime}$ one has:

$$
\tilde{\ell}_{x x^{\prime}}=\int_{\min \left(x, x^{\prime}\right)}^{\max \left(x, x^{\prime}\right)} \frac{\mathrm{d} \eta}{\sqrt{1-D(\eta)}} \geq \ell_{x x^{\prime}}
$$

where $\ell_{x x^{\prime}}=\ell_{x}\left(x^{\prime}\right)=\left|x-x^{\prime}\right| \geq 0$ is the Euclidean distance between $x$ and $x^{\prime}$ (i.e., the interaction distance that is considered in INL models) and $\min (\cdot) / \max (\cdot)$ are the standard minimum/maximum operators.

In the framework of the Finite Element Method (FEM), one, therefore, has to compute interaction distances between integration points where damage is evaluated. A simple trapezoidal rule was used by Desmorat et al. [9] to calculate integral (3), under the assumption that the damage field is constant between adjacent integration points. More recently, Jirásek and Desmorat [19] proposed to use a slightly modified integration rule based on assuming that the damage field is linear between adjacent integration points. According to these authors, such a choice leads to improved accuracy in integral computation and makes the damage process more gradual, making it easier to follow the structural response when damage localization occurs.

Based on a localization problem into a bar submitted to tensile loading, in this short communication, we show that such a choice induces parasite damage diffusion, leading to loose one of the main advantages of ENL formulations. This makes the use of a standard trapezoidal rule more effective. Moreover, the structural response in the final phases of the test can be described by using a dissipative path-following method based on controlling the non-local strain variation of the localizing element instead of the local strain (as in the articles cited above).

\section{Eikonal non-local damage model}

Let us consider a $1 \mathrm{D}$ body occupying all points $x \in \Omega=$ ] $0, L[$. The mechanical behavior of its constituting material is described through an isotropic continuum damage model [21] with a single scalar variable $D$. Accordingly, the Cauchy's stress reads $\sigma=\sigma(x, t)=(1-D) E \varepsilon$, where $E=E(x)$ is the Young's modulus, $\varepsilon=\varepsilon(x, t)=\mathrm{d} u / \mathrm{d} x$ is the infinitesimal strain and $u=u(x, t)$ is the displacement field.

Non-local damage evolution is supposed to occur according to the law, $D=g(\kappa)$, where $\kappa=\kappa(x, t)$ is an internal variable. Its evolution has to fulfill the following non-local Kuhn-Tucker and consistency conditions: $\bar{f} \leq 0, \dot{\kappa} \geq 0, \bar{f} \dot{\kappa}=0$ and $\dot{\bar{f}} \dot{\kappa}=0$. In previous definitions, $\bar{f}=\bar{f}(\sigma, \varepsilon, D)$ is a damage activation function, and symbol $\bullet$ denotes the rate of variation of the quantity $\bullet$. We chose $\bar{f}=\bar{\varepsilon}-\kappa$, where $\bar{\varepsilon}=\bar{\varepsilon}(x, t)$ denotes the non-local strain. Alternatively, instead of $\bar{\varepsilon}$, one can define $\bar{f}$ in terms of the non-local counterparts of the thermodynamic force associated with damage $Y=Y(x, t)=E \varepsilon^{2} / 2$ [24] or other quantities [18].

\subsection{Integral nonlocal (INL) formulation}

In standard INL-type formulations [31], field $\bar{\varepsilon}$ is computed by weighted averaging of field $\varepsilon$ over the entire domain:

$$
\bar{\varepsilon}=\bar{\varepsilon}(x, t)=\frac{\int_{\Omega} \phi\left(\xi_{x x^{\prime}}\right) \varepsilon\left(x^{\prime}, t\right) \mathrm{d} x^{\prime}}{\int_{\Omega} \phi(\xi) \mathrm{d} x^{\prime}}
$$

where $\xi_{x x^{\prime}}=\xi\left(x, x^{\prime}\right)$ denotes the ratio of the interaction distance $\ell_{x x^{\prime}}$ to the characteristic length $\left(\ell_{c}>0\right)$, and $\phi\left(\xi_{x x^{\prime}}\right)$ is a positive monotonic decreasing function of $\xi_{x x^{\prime}}$. The latter function is chosen such that $\phi\left(\xi_{x x^{\prime}}\right)=1$ for $\xi_{x x^{\prime}}=0$, and $\phi\left(\xi_{x x^{\prime}}\right) \rightarrow 0$ when $\xi_{x x^{\prime}}$ increases. A common choice to express $\phi\left(\xi_{x x^{\prime}}\right)$ consists in using the Gaussian distribution function, i.e., $\phi\left(\xi_{x x^{\prime}}\right)=\exp \left(-4 \xi_{x x^{\prime}}^{2}\right)$.

\subsection{Eikonal non-local (ENL) formulation}

According to the ENL approach, the non-local averaging process is the same as in the standard INL formulations. However, non-local interactions change according to the damage field evolution through effective distances. In a 1D setting, the length ratio $\xi_{x x^{\prime}}$ appearing in eq. (4) thus reads:

$$
\xi_{x x^{\prime}}=\frac{\tilde{\ell}_{x x^{\prime}}}{\ell_{c}} \geq \frac{\left|x-x^{\prime}\right|}{\ell_{c}}
$$

According to eqs. (3) and (5), $\tilde{\ell}_{x x^{\prime}}=\left|x-x^{\prime}\right|$ when $D(\eta)=0$ $\forall \eta \in\left[x, x^{\prime}\right]$ (i.e., the standard INL formulation is recovered), whereas $\tilde{\ell}_{x x^{\prime}}>\left|x-x^{\prime}\right|$ when damage occurs (i.e., non-local interactions are reduced). When damage tends to unity for at least 
F. Thierry, G. Rastiello, C. Giry, F. Gatuingt / Mech. Res. Comm. / One-dimensional Eikonal Non-Local (ENL) damage models: Influence of the integration rule for computing interaction distances and indirect loading control on damage localization

one point $\eta^{\prime} \in\left[x, x^{\prime}\right]$, distance $\tilde{\ell}_{x x^{\prime}} \rightarrow+\infty$ and no more nonlocal interactions are possible between the considered material points. Despite what is done in standard INL formulations, nonlocal interactions between material points across highly damaged bands are no more possible. As a consequence, parasite damage diffusion problems that are typically encountered when considering fixed distances can be avoided in a natural way.

\subsection{Numerical formulation in $1 D$}

Consider a domain $\Omega$ that is discretized through a FE mesh $\left(\Omega^{h}\right)$ comprising an odd number $\left(n_{\mathrm{el}}\right)$ of FEs of equal size $(h=$ $\left.L / n_{\mathrm{el}}\right)$.

A continuous linear interpolation is chosen for the unknown displacement field. Integration points (one per element) are identified according to the ordered sequence $\mathcal{I}=\left\{x_{i}\right\}_{i=1}^{n_{\mathrm{el}}}$. The discretized damage field $\left\{D_{i}\right\}_{i=1}^{n_{\mathrm{el}}}=\left\{D\left(x_{i}\right)\right\}_{i=1}^{n_{\mathrm{el}}}$, is evaluated at integration points according to an explicit procedure.

Interaction distances $\left(\tilde{\ell}_{i, j}\right)$ between points $x_{i}$ and $x_{j} \in \mathcal{I}$ are computed at the beginning of each time step based on the last converged damage field (at time $t_{n}$ ), $\left\{D_{i}^{n}\right\}_{i=1}^{n_{\mathrm{el}}}$. They are then used to update the non-local strain field (4) driving damage evolution and kept constant until convergence of the global equilibrium problem (at time $t_{n+1}$ ).

\subsection{Interaction distances computation}

As illustrated by Jirásek and Desmorat [19], different integration techniques can be used for computing eq. (3), and might influence the obtained results:

(i) the first and natural choice consists in assuming that function $\sqrt{1-D}$ is element-wise constant, and performing integration according to a trapezoidal rule. The effective distance $\tilde{\ell}_{i, j}$ between points $x_{i}$ and $x_{j}>x_{i}$ thus reads:

$$
\tilde{\ell}_{i, j}=\tilde{\ell}_{i, j-1}+\frac{h}{2}\left(\frac{1}{\sqrt{1-D_{j-1}}}+\frac{1}{\sqrt{1-D_{j}}}\right)
$$

where $\tilde{\ell}_{i, j-1}$ is the effective distance between $x_{i}$ and $x_{j-1}$;

(ii) an alternative way for computing interaction distance was recently proposed in [19]. Assuming that the damage field is linear between adjacent integration points, distance $\tilde{\ell}_{i, j}$ reads:

$$
\tilde{\ell}_{i, j}=\tilde{\ell}_{i, j-1}+\frac{2 h}{\sqrt{1-D_{j-1}}+\sqrt{1-D_{j}}}
$$

According to the cited work, this formulation shows improved performance from the integration error viewpoint, in particular when damage localization occurs. The influence of such a choice on the damage localization process has not been studied yet, however.

In the following, the ENL formulation with trapezoidal rule (eq. (6)) is denoted ENL1, whereas the other one (eq. (7)) is named ENL2. For considerations holding for both methods, we will use the generic term ENL.

\section{Considered problem}

We consider a bar of length $L$ and constant transverse crosssection $S$ submitted to tensile loading. Displacement is blocked on the left side of the bar $(x=0)$, whereas an indirectly controlled force is imposed at the right extremity $(x=L)$ to ensure describing structural responses characterized by snap-back phases.

Different FE meshes are considered ( $n_{\mathrm{el}}$ ranging from 41 to 2001 linear Bar elements). Damage initiation is forced to occur on the central/weak element (the corresponding integration point denoted $x_{w}$, with $\left.w=\left(n_{\mathrm{el}}+1\right) / 2\right)$. For this purpose, the Young's modulus is spatially distributed as $E_{i}=$ $E\left(x_{i}\right)=E_{0}\left(1-\alpha_{i} / 10\right)$, where $E_{0}$ is a reference Young's modulus, and $\alpha_{i}=\alpha\left(x_{i}\right)$ is chosen as a Gaussian function. centered on $x_{w}$ and with length parameter equal to $L / 10$, i.e., $\alpha_{i}=\exp \left[-400\left(x_{i}-x_{w}\right)^{2} / L^{2}\right]$. This allows for removing the small differences in the structural response (stiffness and load peak) that are observed between different meshes when localization is induced by slightly reducing $E\left(x_{w}\right)$ only.

Damage evolves according to the exponential law $D_{i}=g\left(\kappa_{i}\right)=$ $1-\left(\kappa_{0} / \kappa_{i}\right) \exp \left(-\left\langle\kappa_{i}-\kappa_{0}\right\rangle /\left(\kappa_{c}-\kappa_{0}\right)\right)$, where $\kappa_{i}=\kappa\left(x_{i}\right)$, $\kappa_{0}$ is the non-local strain level corresponding to damage activation, $\kappa_{c}$ is the non-local strain level controlling the shape of the damage evolution function and $\langle\bullet\rangle$ denotes the Macaulay operator (given a real number $\bullet,\langle\bullet\rangle=\bullet$ if $\bullet \geq 0$ and $\langle\bullet\rangle=0$ otherwise). Different evolution models (linear, bi-linear, exponential...) could have alternatively be used without substantially changing the theoretical formulation and its numerical implementation.

A quasi-newton method is used to solve the global equilibrium problem. To follow the structural response in the snap-back phase, the external loading is controlled indirectly based on a simple path-following technique [8, 34].

Two path-following constraint equations are considered:

(i) the first one imposes the local strain variation of the weak element $\left(\Delta \varepsilon_{w}-\Delta \tau=0\right.$, with $\Delta \tau>0$ a user-defined path-step length) between two successive loading steps, as in $[9,19]$;

(ii) the second one imposes the non-local strain variation of the weak element $\left(\Delta \bar{\varepsilon}_{w}-\Delta \tau=0, \Delta \tau>0\right)$. Given the simple considered geometrical and mechanical models, the chosen constraint ensures achieving dissipative responses only since the maximum non-local strain variation is always attained at the weak element.

More details concerning the numerical solution procedure are given in Appendix A.

\section{Results and discussion}

\subsection{Global responses}

Let us first consider a finite element mesh with 81 Bar FEs and compare force-displacement $\left(F, u_{L}\right)$ responses obtained by 
F. Thierry, G. Rastiello, C. Giry, F. Gatuingt / Mech. Res. Comm. / One-dimensional Eikonal Non-Local (ENL) damage models: Influence of the integration rule for computing interaction distances and indirect loading control on damage localization

considering the local, INL and ENL damage models (fig. 1), for both integration formulas introduced in section 2.4 .

As already observed by Rastiello et al. [33], ENL and INL models provide very similar responses for moderate damage levels (non-local interactions are mainly controlled by Euclidean distances in this phase). Then, ENL responses diverge from the INL one and, as expected, tend to the local one when damage grows up to unity on the weak element $\left(D_{w} \rightarrow 1\right)$. The ENL1 and ENL2 formulations give almost identical results until very high damage levels (approximately $D_{w} \approx 0.9$ ), then they exhibit some differences. In particular, the ENL2 formulation provides a smoother failure of mechanical strength than ENL1 and a higher energy dissipation. In both cases, however:

(i) controlling the simulation through imposing $\Delta \bar{\varepsilon}_{w}$ (solid lines in fig. 1) makes it possible to properly follow the whole damage localization process;

(ii) conversely, using a constraint equation based on $\Delta \varepsilon_{w}$ (dashed lines in fig. 1) - or the relative displacement of the nodes of the weak element as in $[33,19]$ - makes it very difficult to control the simulation when $D_{w} \rightarrow 1$, in particular for the ENL1 formulation. And this is independent of the chosen $\Delta \tau$. By the way, this problem partly motivated Jirásek and Desmorat [19] to replace the trapezoidal integration rule by eq. (7). Notice that for the considered mesh no convergence issues were experienced; however, this can append for finer meshes (e.g., for $n_{\mathrm{el}}=161$ or higher). See Appendix A for more details.

From here-forth all results are obtained using the constraint equation based on the non-local strain variation. Some additional explanations concerning this choice will be given in the following.

\subsection{Damage localization}

As illustrated by the damage distributions along the bar (fig. 2), both ENL implementations model a progressive damage localization in the central region of the bar.

Based on these results, at any pseudo-time, one can identify three different zones in the bar: (i) an undamaged region $\left(\mathcal{I}^{\text {elastic }}\right)$, where the material response stays elastic and no damage occurs; (ii) an inactive damage region $\left(\mathcal{I}^{\text {inactive }}\right)$, where material points are damaged but no more experience damage evolution; (iii) an active damage region $\left(\mathcal{I}^{\text {active }}\right)$, where damaging is in progress.

During the fist phases of the test, region $\mathcal{I}^{\text {active }}$ grows up and $\mathcal{I}^{\text {elastic }}$ shrinks, whereas $\mathcal{I}^{\text {inactive }}$ is empty. Then, due to the evolution of interaction distances, points belonging to $\mathcal{I}^{\text {active }}$ progressively move to $\mathcal{I}^{\text {inactive }}$, while $\mathcal{I}^{\text {elastic }}$ stay constant (i.e., the size of the damaged band does not change).

To better understand such a damage/strain localization process, fig. 3 depicts the evolution of damage and local strain at $x_{w}$ and $x_{w+1}$, with respect to $\bar{\epsilon}_{w}$. Since this quantity is controlled

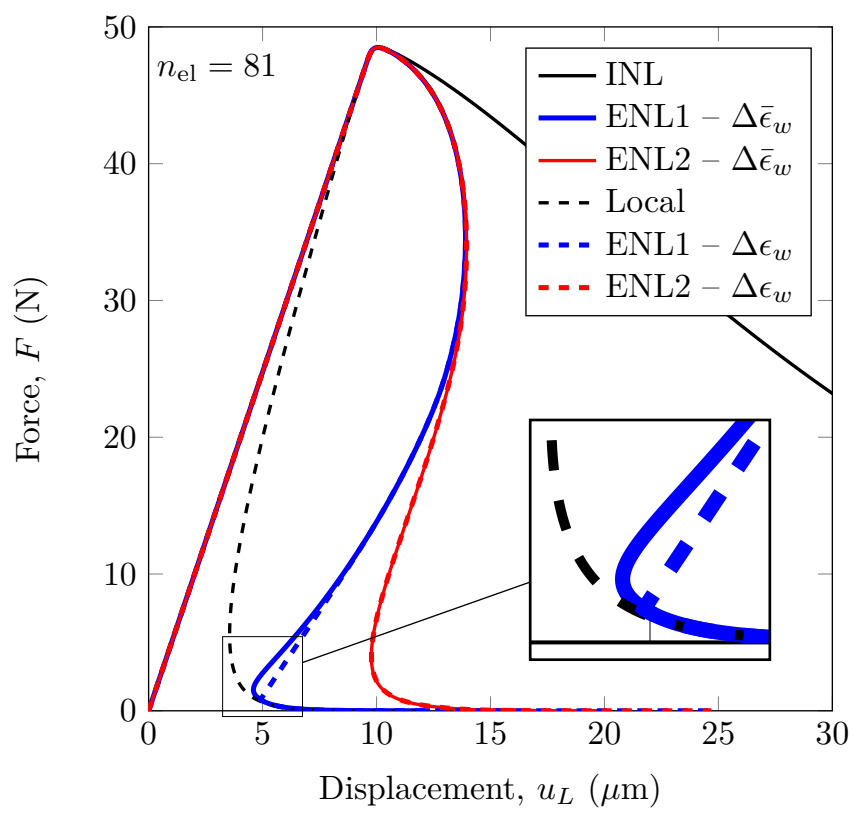

Figure 1: ENL formulations 1 and 2 - Force-displacement responses obtained using local, INL and ENL damage models (finite element mesh with 81 elements). Results obtained controlling the simulation on the non-local strain variation of the weak element and on the local strain variation of the weak element - Numerical parameters: $L=100 \mathrm{~mm}, S=5 \cdot 10^{-3} \mathrm{~m}^{2}$, $E=100 \mathrm{MPa}, \kappa_{0}=10^{-4}, \kappa_{c}=10 \kappa_{0}, \ell_{c}=L / 5=20 \mathrm{~mm}$, $\Delta \tau=\kappa_{0} / 50$ (for both the constraint equations)

during the computation, such representation also provides the pseudo-time evolution of the considered variables.

As already mentioned, despite what is provided by INL formulations, during the localization process, damage progressively tends to unity on the weak element (solid lines, fig. 3a) for both ENL models. Some differences between ENL1 and ENL2 formulations are, however, clear. In particular:

(i) the ENL1 formulation clearly models strain localization. Damage is equal to unity in the central element, whereas it remains constant from a certain instant forward in the neighboring element (dashed blue line). Simultaneously, the non-local strain progressively tends to the local one in the weak element, while it gradually decreases in the neighboring element. As expected, such a process is accompanied by the elastic unloading of the neighboring element, which progressively tends to be undeformed.

It is worth noticing that, during the final damage localization phases, $\epsilon_{w}$ decreases to ensure a gradual/smooth increase of $\bar{\epsilon}_{w}$ and thus of $D_{w}$. This response clearly illustrates why a path-following constraint equation based on controlling $\Delta \epsilon_{w}$ cannot properly work. Indeed, since $\epsilon_{w}$ is not allowed to decrease in that case, $\bar{\epsilon}_{w}$ abruptly increases, leading to a rapid/uncontrolled increase in $D_{w}$. This explains the jump (dashed blue line) observed in the global response depicted in fig. 1; 
F. Thierry, G. Rastiello, C. Giry, F. Gatuingt / Mech. Res. Comm. / One-dimensional Eikonal Non-Local (ENL) damage models: Influence of the integration rule for computing interaction distances and indirect loading control on damage localization

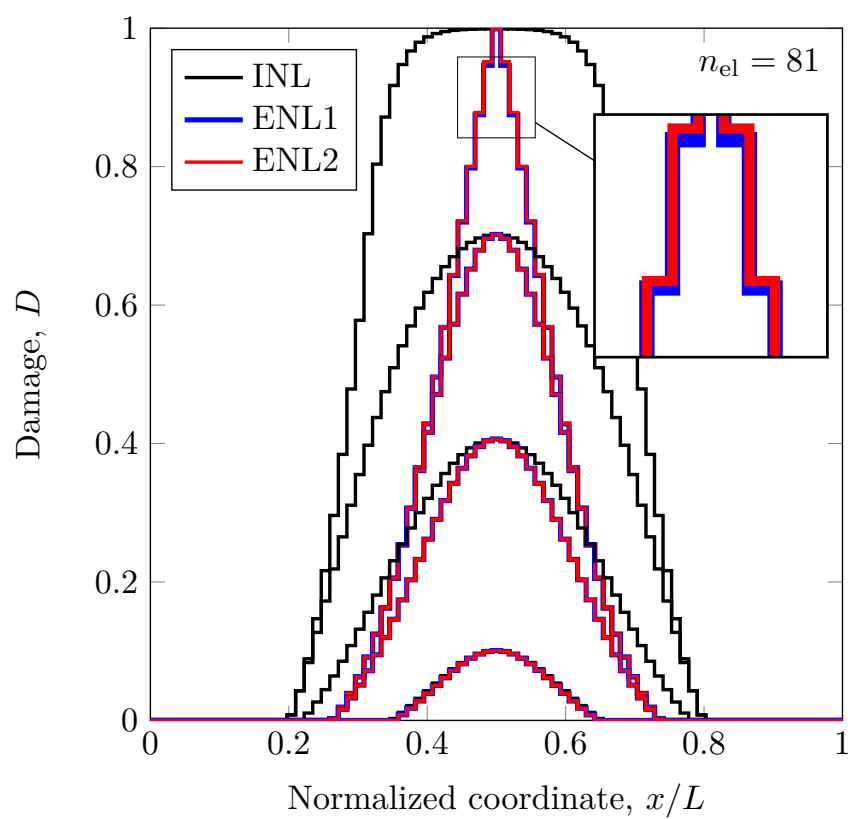

Figure 2: ENL formulations 1 and 2 - Damage distributions along the bar at different time-steps (81 finite elements).

(ii) conversely, when using the ENL2 formulation, damage in the neighboring elements continue to increase. Moreover, $\bar{\epsilon}_{w}$ never reaches $\epsilon_{w}$. This ensures more numerical robustness for the ENL2 formulation, as strain localization in only one element is significantly delayed.

This behavior can be easily explained in light of eq. (7), considering three neighboring integration points $\left(x_{w}, x_{w+1}, x_{w+2}\right)$ such that $D_{w+2}<D_{w+1}<D_{w}$ and $D_{w} \rightarrow 1^{-}$(same considerations hold for $\left(x_{w}, x_{w-1}, x_{w-2}\right)$ due to symmetry). Under these conditions, one can easily show that:

(i) in the case of the ENL1 formulation, condition $D_{w} \rightarrow$ $1^{-}$implies that $\tilde{\ell}_{w, w+1}$ can become larger than the maximum length such that $\phi\left(\tilde{\ell}_{w, w+1}\right)$ becomes very small (negligible):

$$
\tilde{\ell}_{w, w+1} \approx \frac{h}{2 \sqrt{1-D_{w}}} \gg \beta \ell_{c}
$$

with $\beta \approx 1.5$ for the Gaussian weighting function. As a consequence, point $x_{w+1}$ (as well as any other integration point) no more interacts with $x_{w}$, and damage evolution cannot occur in the bar;

(ii) on the contrary, when $D_{w} \rightarrow 1^{-}$, the interaction distance $\tilde{\ell}_{w, w+1}$ that is considered in the ENL2 formulation can still be smaller than $\beta \ell_{c}$ :

$$
\tilde{\ell}_{w, w+1} \approx \frac{2 h}{\sqrt{1-D_{w+1}}} \leq \beta \ell_{c}
$$

if $D_{w+1}$ is small enough (compared to unity). As a consequence, $D_{w+1}$ can increase due to the increasing strain at $x_{w}$ (this term still contribute to the non-local averaged strain at $\left.x_{w+1}\right)$.
From a theoretical point of view, the only way for preventing non-local interactions between $x_{w}$ and other points to occur, is that also $D_{w+1} \rightarrow 1^{-}$. Indeed, since $\tilde{\ell}_{w+1, w+2}=$ $2 h /\left(\sqrt{1-D_{w+1}}+\sqrt{1-D_{w+2}}\right)$, one can show that:

$$
\tilde{\ell}_{w, w+2}=\tilde{\ell}_{w, w+1}+\tilde{\ell}_{w+1, w+2} \leq \beta \ell_{c}
$$

as long as $D_{w+1}$ and $D_{w+2}$ are small enough. Once condition $D_{w+1} \rightarrow 1^{-}$is achieved, $\tilde{\ell}_{w, w+1}$ and $\tilde{\ell}_{w+1, w+2}$ both become very large:

$$
\begin{aligned}
\tilde{\ell}_{w, w+1} & \gg \beta \ell_{c} \\
\tilde{\ell}_{w+1, w+2} & \approx \frac{2 h}{\sqrt{1-D_{w+1}}} \gg \beta \ell_{c}
\end{aligned}
$$

and no more interactions are possible between points $x_{w}$, $x_{w+1}$ and their neighbors.

From a numerical viewpoint, such a condition is however attained when $\epsilon_{w} \rightarrow+\infty$ (i.e., for very high sample elongation levels). Notice that when $\epsilon_{w} \approx 1=10^{4} \times$ $\epsilon_{0}, D_{w+1}$ is still close to 0.99 for the ENL2 formulation. Such a damage evolution can be limited numerically through considering different weighting functions (e.g., the bell-shaped polynomial function) such that $\phi=0$ for $\tilde{\ell}_{w, w+1} \geq \ell_{c}$. In that case, the maximum attainable damage value at $x_{w+1}$ will depend on the mesh size (i.e., smaller $h$ higher $D_{w+1}$ ).

Some additional considerations have to be provided concerning the fact that, when using the ENL1 formulation, $\varepsilon_{w}$ tends to reduce during the intermediate phases of the test, whereas $\bar{\varepsilon}_{w}$ is still increasing (imposed quantity). This response can be easily explained keeping in mind that the non-local strain variation at $x_{w}$ between $t_{n}$ and $t_{n+1}$, can be written as:

$$
\Delta \bar{\varepsilon}_{w}=\frac{\sum_{j}\left(\varepsilon_{j}^{n}+\Delta \varepsilon_{j}\right) \phi_{w, j}^{n}}{\sum_{j} \phi_{w, j}^{n}}-\frac{\sum_{j} \varepsilon_{j}^{n} \phi_{w, j}^{n-1}}{\sum_{j} \phi_{w, j}^{n-1}}=\Delta \tau
$$

where $\varepsilon_{j}^{n}=\varepsilon^{n}\left(x_{j}\right)$ is the strain at $x_{j}$ and time $t_{n}$ and, given the chosen explicit procedure for updating interaction distances, $\phi_{w, j}^{n}$ and $\phi_{w, j}^{n-1}$ are the weighting functions corresponding to damage distributions at $t_{n}$ and $t_{n-1}$. After some algebraic manipulations, eq. (13) can also be written as:

$$
\Delta \bar{\varepsilon}_{w}=\Delta \varepsilon_{w}^{*}+\Delta \bar{\varepsilon}_{w}^{*}+\varepsilon_{w}^{*}+\bar{\varepsilon}_{w}^{*}=\Delta \tau
$$

with:

$$
\begin{aligned}
\Delta \varepsilon_{w}^{*} & =\frac{\Delta \varepsilon_{w}}{\sum_{j} \phi_{w, j}^{n}} \\
\Delta \bar{\varepsilon}_{w}^{*} & =\frac{\sum_{j \neq w} \Delta \varepsilon_{j} \phi_{w, j}^{n}}{\sum_{j} \phi_{w, j}^{n}} \\
\varepsilon_{w}^{*} & =\varepsilon_{w}^{n} \frac{\sum_{j} \phi_{w, j}^{n-1}-\sum_{j} \phi_{w, j}^{n}}{\left(\sum_{j} \phi_{w, j}^{n}\right)\left(\sum_{j} \phi_{w, j}^{n-1}\right)} \\
\bar{\varepsilon}_{w}^{*} & =\frac{\sum_{j \neq w} \varepsilon_{j}^{n} \phi_{w, j}^{n}}{\sum_{j} \phi_{w, j}^{n}}-\frac{\sum_{j \neq w} \varepsilon_{j}^{n} \phi_{w, j}^{n-1}}{\sum_{j} \phi_{w, j}^{n-1}}
\end{aligned}
$$


F. Thierry, G. Rastiello, C. Giry, F. Gatuingt / Mech. Res. Comm. / One-dimensional Eikonal Non-Local (ENL) damage models: Influence of the integration rule for computing interaction distances and indirect loading control on damage localization

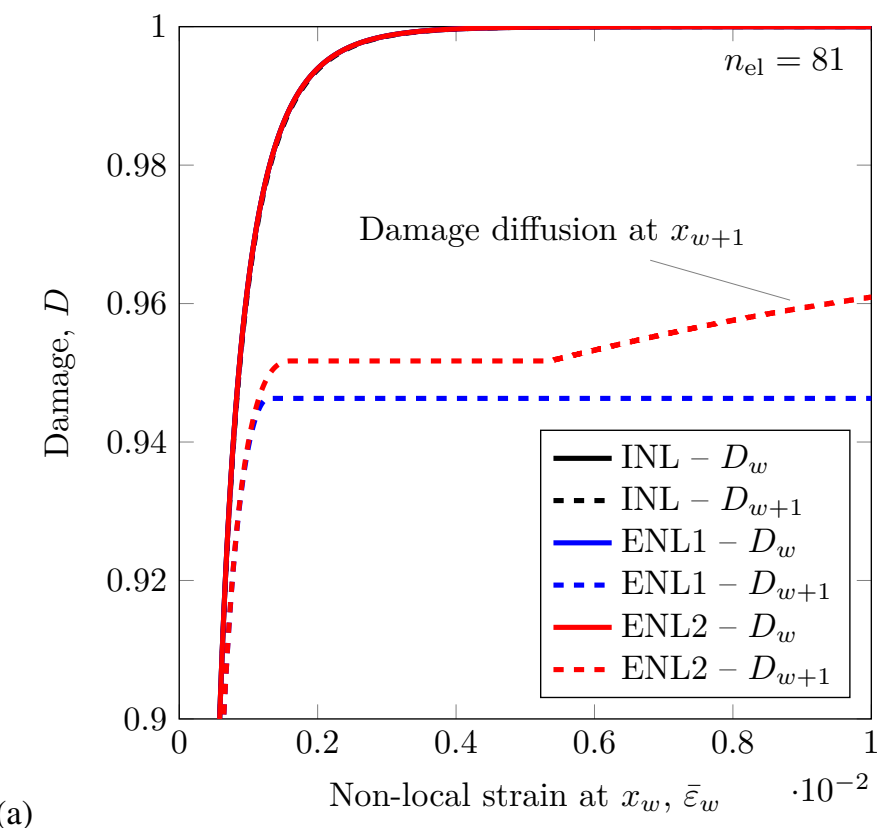

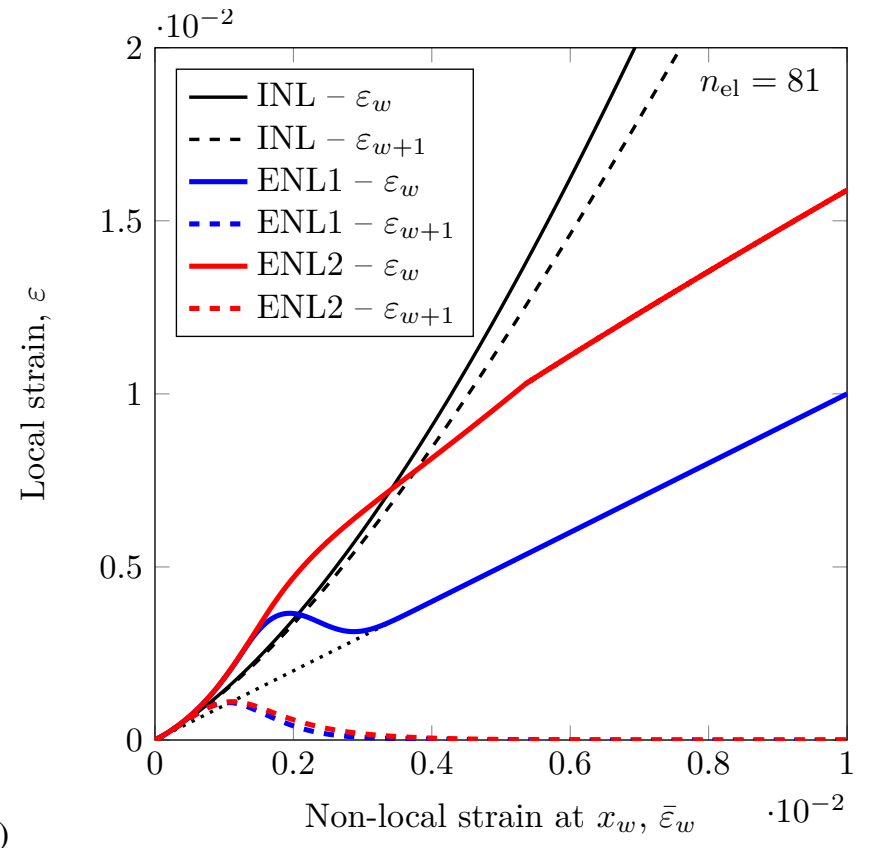

Figure 3: ENL formulations — Damage (a) and non-local strain (b) evolution at $x_{w}$ and $x_{w+1}$ (81 finite elements).

The evolution of contributions appearing in eq. (14) is depicted in fig. 4. When damage occurs, integration points $x_{j} \in \mathcal{I}$ that are far enough from $x_{w}$ start experiencing negative local strain variations. As a consequence, $\Delta \bar{\varepsilon}_{w}^{*}$ start decreasing, while other contributions still increase in order to ensure the fulfillment of constraint (14). During damage localization, the number of integration points experiencing negative strain variations increases, leading to $\Delta \bar{\varepsilon}_{w}^{*}<0$. In the meanwhile, points $x_{j} \neq$ $x_{w}$ progressively move from $\mathcal{I}^{\text {active }}$ to $\mathcal{I}^{\text {inactive }}$. During this phase, contribution $\bar{\varepsilon}_{w}^{*}+\varepsilon_{w}^{*}$ increases (it is mainly driven by $\left.\varepsilon_{w}\right)$ and become larger than $\Delta \tau$. Then, since $\varepsilon_{w}$ has become quite large, and negative contributions coming from neighbor points progressively vanish, fulfilling condition (14) implies that $\Delta \varepsilon_{w}^{*}<0$. The local strain at $x_{w}$ is thus reduced. Once the strain localization is complete, $\Delta \bar{\varepsilon}_{w}=\Delta \varepsilon_{w}^{*}=\Delta \varepsilon_{w}=\Delta \tau>$ 0 , whereas all the remaining contributions become negligible, because $\sum_{j} \phi_{w, j}^{n-1}=\sum_{j} \phi_{w, j}^{n}=1$ and $\sum_{j \neq w} \Delta \varepsilon_{j} \phi_{w, j}^{n}=$ $\sum_{j \neq w} \varepsilon_{j}^{n} \phi_{w, j}^{n}=\sum_{j \neq w} \varepsilon_{j}^{n} \phi_{w, j}^{n-1} \approx 0$.

\subsection{Parasite/residual mesh-dependency}

Features mentioned above make ENL formulations suitable for representing localization processes characterized by a progressive transition from diffuse damage to fracture (e.g., cracking of quasi-brittle materials). The observed tendency toward strain localization is responsible, however, of the small differences that are observed in the global structural responses computed for different finite element meshes for high damage levels (fig. 5).

For both ENL formulations, when non-local interactions between the weak element and its neighbors vanish (or are lower than a critical value), damage evolution becomes local. The energy still available at the weak element is consumed in a local manner [33]. In other words, regularization properties are lost, and parasite mesh dependency issues are observed.

As a consequence, in addition to inducing damage diffusion for high elongation levels, the ENL2 formulation only slightly helps in improving such a feature/limitation which is proper to other non-local damage models with evolving interaction distances (see e.g., [14]).

\section{Conclusive remarks}

ENL damage formulations exhibit good regularization properties and naturally allow one to model a progressive strain localization and damage-to-fracture transition [9, 33, 19].

Focusing on a 1D formulation, in this contribution, we analyzed the effect of different distances integration techniques to compute interaction distances between points from solving a damage-dependent Eikonal problem. In particular, we pointed out that parasite damage diffusion can occur when assuming that the damage field is element-wise linear in the metric function [19]. Conversely, this is not the case when considering that damage is element-wise constant $[9,33]$.

Moreover, controlling the simulation through the local strain variation of the element where damage localization occurs, does not allow to simulate a smooth damage evolution. Conversely, this result can be achieved by using a constraint equation based on the non-local strain variation. Once this choice is made, we showed that the localizing element's local strain has to decrease during the final localization phases to ensure a gradual evolution of the non-local strain and, thus, a smooth damage evolution. 
F. Thierry, G. Rastiello, C. Giry, F. Gatuingt / Mech. Res. Comm. / One-dimensional Eikonal Non-Local (ENL) damage models: Influence of the integration rule for computing interaction distances and indirect loading control on damage localization

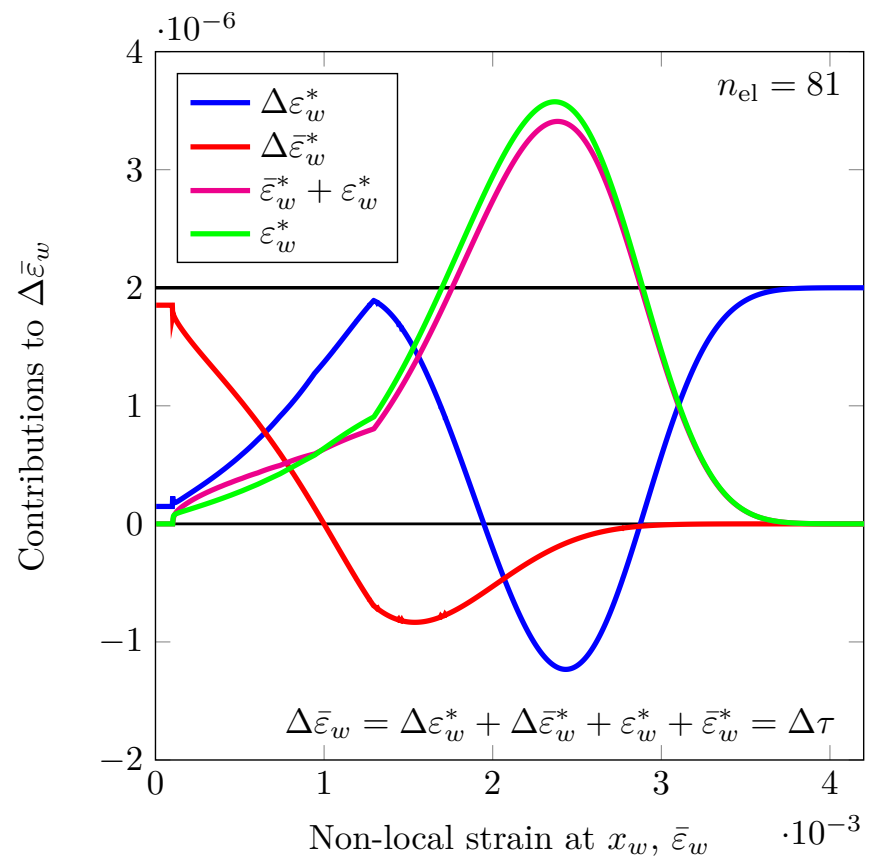

Figure 4: ENL formulation 1 - Contributions to the non-local strain variation (14) at the weak element (finite element mesh comprising 81 elements).

\section{Appendix A. Solving method}

A path-following method is used to solve the non-linear equilibrium problem. Accordingly, the external force vector is written as $\mathbf{f}_{\text {ext }}=\lambda \hat{\mathbf{f}}$, where the loading factor $\lambda \in \mathbb{R}$ is a novel problem unknown and $\hat{\mathbf{f}}$ is a constant vector providing the direction of the loading.

The problem to be solved consists of computing the nodal displacements vector $(\mathbf{d})$ and the loading factor $(\lambda)$ that fulfill the equilibrium equation, $\mathbf{r}\left(\mathbf{d}, \lambda ;\left\{D_{i}\right\}_{i=1}^{n_{\mathrm{el}}}\right)=\mathbf{f}^{\mathrm{int}}\left(\mathbf{d} ;\left\{D_{i}\right\}_{i=1}^{n_{\mathrm{el}}}\right)-$ $\mathbf{f}^{\operatorname{ext}}(\lambda)=\mathbf{0}$ (with $\mathbf{r}$ denoting the residual and $\mathbf{f}^{\text {int }}$ being the internal forces vector), and a path-following constraint equation, $p(\mathbf{d}, \lambda ; \Delta \tau)=0$.

The unknown nodal displacement variation vector $\delta \mathbf{d}^{k+1}$ at the $k+1$-th iteration is decomposed as [35, 7, 8]:

$$
\delta \mathbf{d}^{k+1}=\delta \lambda^{k+1} \delta \mathbf{d}_{\mathrm{I}}^{k+1}+\delta \mathbf{d}_{\mathrm{II}}^{k+1}
$$

where $\delta \mathbf{d}_{\mathrm{I}}^{k+1}$ and $\delta \mathbf{d}_{\mathrm{II}}^{k+1}$ are solved from:

$$
\mathbf{K}^{k} \delta \mathbf{d}_{\mathrm{I}}^{k+1}=\hat{\mathbf{f}} \quad \mathbf{K}^{k} \delta \mathbf{d}_{\mathrm{II}}^{k+1}=-\mathbf{r}^{k}
$$

with $\mathbf{K}^{k}=\mathbf{K}^{k}\left(\mathbf{d}^{k} ;\left\{D_{i}^{k}\right\}_{i=1}^{n_{\mathrm{el}}}\right)$ denoting the global secant stiffness matrix at iteration $k$ and $\mathbf{r}^{k}=\mathbf{r}^{k}\left(\mathbf{d}^{k}, \lambda^{k} ;\left\{D_{i}^{k}\right\}_{i=1}^{n_{\mathrm{el}}}\right)$ the residual vector.

The resolution is performed in two steps. Displacement variations $\left(\delta \mathbf{d}_{\mathrm{I}}^{k+1}, \delta \mathbf{d}_{\mathrm{II}}^{k+1}\right)$ are first computed. Then the loading factor increment $\delta \lambda^{k+1}$ is computed from solving the path-following constraint equation. Details and examples of path-following constraint equations can be found in existing papers (see e.g., [6, 22, 32, 34] among others).
To control the non-local strain variation of the weak element, the constraint equation is written as $p=\Delta \bar{\varepsilon}_{w}^{k+1}-\Delta \tau=0$, where $\Delta \bar{\varepsilon}_{w}^{k+1}$ reads as in eq. (13). Given the additive displacement decomposition (A.1) and the linear relationship between local strains and nodal displacements (through strain - displacement interpolation matrices), previous constraint equation can be easily rewritten as a linear function of $\delta \lambda^{k+1}$. One finally obtains:

$$
\delta \lambda^{k+1}=\frac{\Delta \tau^{*} \sum_{j} \phi_{w, j}^{n}-\sum_{j} \Delta \varepsilon_{j}^{k} \phi_{w, j}^{n}-\sum_{j} \delta \varepsilon_{j, \mathrm{II}}^{k+1} \phi_{w, j}^{n}}{\sum_{j} \delta \varepsilon_{j, \mathrm{I}}^{k+1} \phi_{w, j}^{n}}
$$

where $\Delta \varepsilon_{j}^{k}$ is the local strain variation between iteration $k$ and loading step $n,\left(\delta \bar{\varepsilon}_{j, \mathrm{I}}^{k+1}, \delta \bar{\varepsilon}_{j, \mathrm{II}}^{k+1}\right)$ are the non-local strain variations corresponding to displacement variations (A.2), and:

$$
\Delta \tau^{*}=\Delta \tau-\left(\frac{\sum_{j} \epsilon_{j}^{n} \phi_{w, j}^{n}}{\sum_{j} \phi_{w, j}^{n}}-\frac{\sum_{j} \epsilon_{j}^{n} \phi_{w, j}^{n-1}}{\sum_{j} \phi_{w, j}^{n-1}}\right)
$$

Notice that, when using an INL formulation, $\Delta \tau^{*}=\Delta \tau$, and $\phi_{w, j}^{n}=\phi_{w, j}^{n-1}=\phi_{w, j}$, with $\phi_{w, j}$ being the constant value of the weighting function between points $x_{w}$ and $x_{j}$.

Solving the constraint equation on the strain variation of the weak element, $p=\Delta \varepsilon_{w}^{k+1}-\Delta \tau=0$, is even more simple. In that case, one directly obtains:

$$
\delta \lambda^{k+1}=\frac{\Delta \tau-\Delta \varepsilon_{w}^{k}-\delta \varepsilon_{w, \mathrm{II}}^{k+1}}{\delta \varepsilon_{w, \mathrm{I}}^{k+1}}
$$

Once $\delta \lambda^{k+1}$ is computed, $\mathbf{d}^{k+1}$ and $\lambda^{k+1}$ are updated and the discretized damage field is evaluated according to an explicit procedure. The residual vector is then computed, and convergence checked based on the following error measure, $\operatorname{err}^{k+1}=\left\|\mathbf{r}^{k+1}\right\| /\left(S f_{t}\right)$, with $f_{t}$ denoting the tensile strength of the material.

During computations the stiffness matrix has been updated every 5 global iterations, the Choleski decomposition was used for solving (A.2), and the tolerance was set to $10^{-5}$. All the results depicted in the paper were obtained considering $\Delta \tau=\kappa_{0} / 50$ (for both the constraint equations) in order to ensure a gradual damaging process.

One can show that the constraint equations were strictly fulfilled at each pseudo-time step for all the simulations performed in this study. However, convergence properties are quite different depending on the chosen ENL formulation. In both cases, if one refers to results depicted in fig. 1, the number of global iterations to converge $(m)$ is lower for the constraint equation on $\Delta \bar{\epsilon}_{w}$ ( $m \in[2,12]$ for both the ENL formulations) than for the constraint equation on $\Delta \epsilon_{w}(m \in[2,50]$ for the ENL1 formulation, and $m \in[2,20]$ for the ENL2 model). Notice that for the mesh considered in fig. $1\left(n_{\mathrm{el}}=81\right)$, convergence is always achieved, but for finer meshes (e.g., for $n_{\mathrm{el}}=161$ ) controlling the simulation through $\Delta \epsilon_{w}$ does not ensure achieving convergence when using the ENL1 formulation. 
F. Thierry, G. Rastiello, C. Giry, F. Gatuingt / Mech. Res. Comm. / One-dimensional Eikonal Non-Local (ENL) damage models: Influence of the integration rule for computing interaction distances and indirect loading control on damage localization

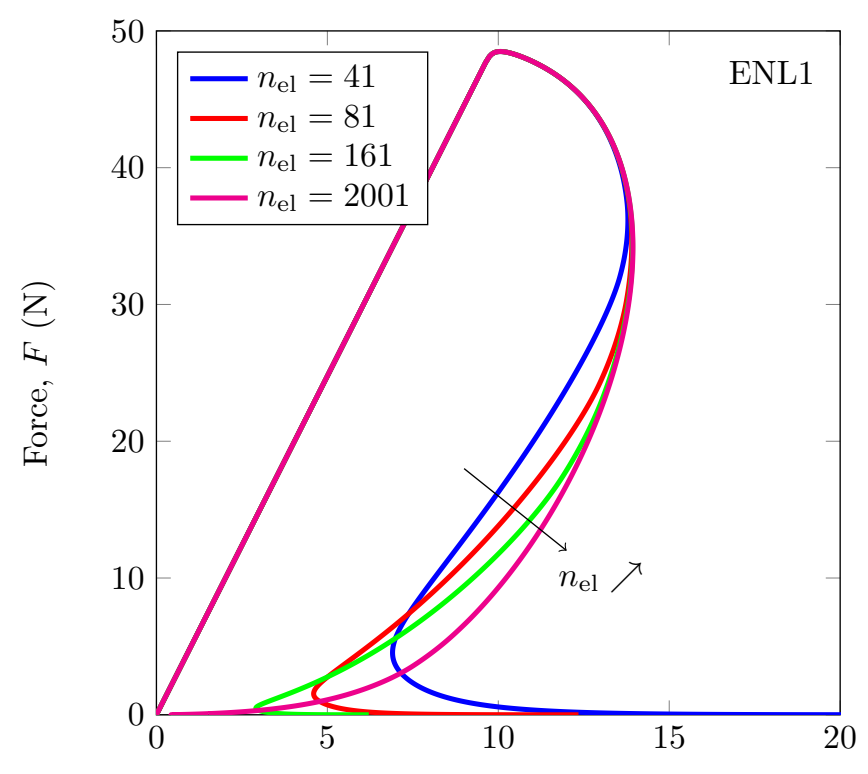

(a)

Displacement, $u_{L}(\mu \mathrm{m})$

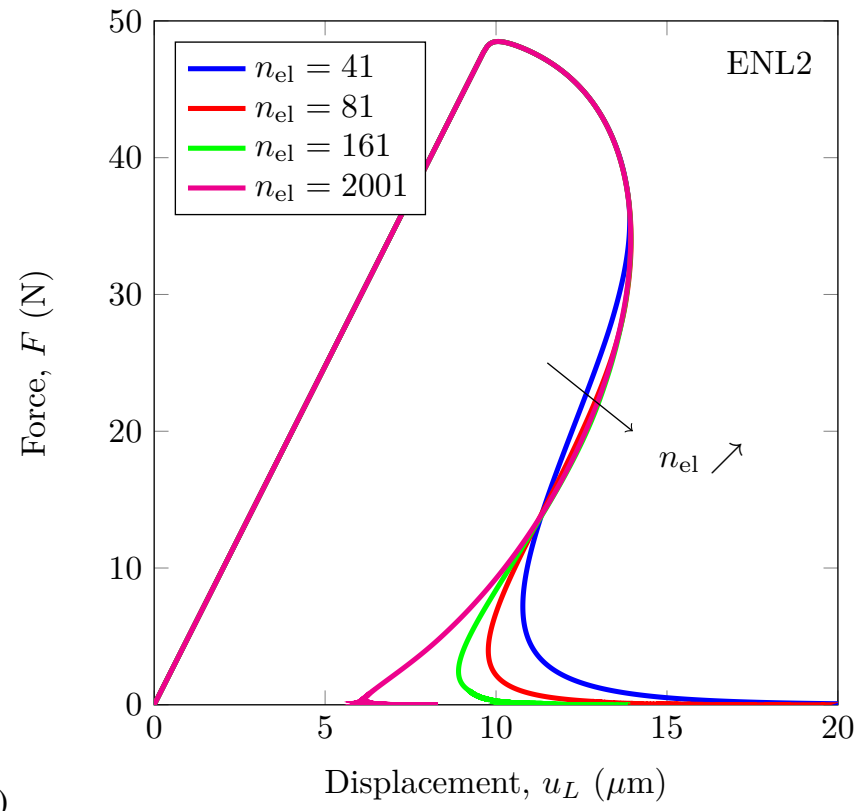

Figure 5: ENL formulations - Force-displacement $\left(F, u_{L}\right)$ responses obtained for different mesh refinement levels (41, 81, 161 and 2001 finite elements) considering the ENL1 (a) and ENL2 (b) formulations. The corresponding element size values range from $\ell_{c} / 8$ to $\ell_{c} / 400$ approximately.

Now, if one refers to results depicted in fig. 5, one can easily show that for the chosen $\Delta \tau$, the number of iteration to converge (at a given non-local strain level) is almost independent of the mesh refinement (for both ENL formulations). Conversely, as expected, there is a tendency to increase the number of iterations when $\Delta \tau$ increases, but such an increase is quite limited and does not strongly affect the convergence features of the solving algorithm.

\section{Acknowledgements}

G. Rastiello was supported by the SEISM Institute (http: //www.institut-seism.fr). The authors declare that they have no known competing financial interests or personal relationships that could have appeared to influence the work reported in this paper.

\section{References}

[1] Z. P. Bažant, J.-L. Le, and C. G. Hoover. Nonlocal boundary layer (nbl) model: overcoming boundary condition problems in strength statistics and fracture analysis of quasibrittle materials. In Proceedings of the 7th International Conference on Fracture Mechanics of Concrete and Concrete Structures, pages 135-143, 2010.

[2] Z. P. Bažant and M. Jirásek. Nonlocal Integral Formulations of Plasticity and Damage: Survey of Progress. Journal of Engineering Mechanics, 128 (11):1119-1149, Nov. 2002. doi: 10.1061/(ASCE)0733-9399(2002)128: 11(1119).

[3] P. Bernard, N. Moës, and N. Chevaugeon. Damage growth modeling using the thick level set (tls) approach: Efficient discretization for quasistatic loadings. Computer Methods in Applied Mechanics and Engineering, 233-236:11 - 27, 2012. ISSN 0045-7825. doi: https://doi.org/10. 1016/j.cma.2012.02.020.
[4] G. Borino, B. Failla, and F. Parrinello. A symmetric nonlocal damage theory. International Journal of Solids and Structures, 40(13):3621-3645, June 2003. ISSN 0020-7683. doi: 10.1016/S0020-7683(03)00144-6.

[5] B. Bourdin, G. A. Francfort, and J.-J. Marigo. The variational approach to fracture. Journal of Elasticity, 91(1-3):5-148, 2008.

[6] Z. Chen and H. L. Schreyer. A numerical solution scheme for softening problems involving total strain control. Computers \& Structures, 37(6): 1043-1050, Jan. 1990. ISSN 0045-7949. doi: 10.1016/0045-7949(90) 90016-U.

[7] M. A. Crisfield. A fast incremental/iterative solution procedure that handles "snap-through". Computers \& Structures, 13(1):55-62, June 1981. ISSN 0045-7949. doi: 10.1016/0045-7949(81)90108-5.

[8] R. de Borst, M. A. Crisfield, J. J. C. Remmers, and C. V. Verhoosel. Nonlinear Finite Element Analysis of Solids and Structures, 2nd Edition, 2012.

[9] R. Desmorat, F. Gatuingt, and M. Jirásek. Nonlocal models with damagedependent interactions motivated by internal time. Engineering Fracture Mechanics, 142:255-275, July 2015. ISSN 0013-7944. doi: 10.1016/j. engfracmech.2015.06.015.

[10] G. A. Francfort and J.-J. Marigo. Revisiting brittle fracture as an energy minimization problem. Journal of the Mechanics and Physics of Solids, 46(8):1319-1342, 1998.

[11] M. Frémond and B. Nedjar. Damage, gradient of damage and principle of virtual power. International Journal of Solids and Structures, 33(8): 1083-1103, Mar. 1996. ISSN 0020-7683. doi: 10.1016/0020-7683(95) 00074-7.

[12] J. F. Ganghoffer, L. J. Sluys, and R. de Borst. A reappraisal of nonlocal mechanics. European Journal of Mechanics - A/Solids, 18(1):17-46, Jan. 1999. ISSN 0997-7538. doi: 10.1016/S0997-7538(99)80002-3.

[13] M. Geers, R. De Borst, W. Brekelmans, and R. Peerlings. Strain-based transient-gradient damage model for failure analyses. Computer methods in applied mechanics and engineering, 160(1-2):133-153, 1998.

[14] C. Giry, F. Dufour, and J. Mazars. Stress-based nonlocal damage model. International Journal of Solids and Structures, 48(25):3431-3443, Dec. 2011. ISSN 0020-7683. doi: 10.1016/j.ijsolstr.2011.08.012.

[15] P. Grassl and M. Jirásek. Damage-plastic model for concrete failure. International Journal of Solids and Structures, 43(22):7166-7196, Nov. 2006. ISSN 0020-7683. doi: 10.1016/j.ijsolstr.2006.06.032.

[16] D. Grégoire, L. B. Rojas-Solano, and G. Pijaudier-Cabot. Failure and size 
F. Thierry, G. Rastiello, C. Giry, F. Gatuingt / Mech. Res. Comm. / One-dimensional Eikonal Non-Local (ENL) damage models: Influence of the integration rule for computing interaction distances and indirect loading control on damage localization

effect for notched and unnotched concrete beams. International Journal for Numerical and Analytical Methods in Geomechanics, 37(10):14341452, 2013.

[17] M. Jirásek, S. Rolshoven, and P. Grassl. Size effect on fracture energy induced by non-locality. International Journal for Numerical and Analytical Methods in Geomechanics, 28(7-8):653-670, 2004.

[18] M. Jirásek. Nonlocal models for damage and fracture: Comparison of approaches. International Journal of Solids and Structures, 35(31): 4133-4145, Nov. 1998. ISSN 0020-7683. doi: 10.1016/S0020-7683(97) 00306-5.

[19] M. Jirásek and R. Desmorat. Localization analysis of nonlocal models with damage-dependent nonlocal interaction. International Journal of Solids and Structures, 174-175:1-17, Nov. 2019. ISSN 0020-7683. doi: 10.1016/j.ijsolstr.2019.06.011.

[20] A. Krayani, G. Pijaudier-Cabot, and F. Dufour. Boundary effect on weight function in nonlocal damage model. Engineering Fracture Mechanics, 76(14):2217-2231, Sept. 2009. ISSN 0013-7944. doi: 10.1016/ j.engfracmech.2009.07.007.

[21] J. Lemaitre and R. Desmorat. Engineering Damage Mechanics: Ductile Creep, Fatigue and Brittle Failures. Springer-Verlag, Berlin Heidelberg, 2005. ISBN 978-3-540-21503-5. doi: 10.1007/b138882.

[22] E. Lorentz and P. Badel. A new path-following constraint for strainsoftening finite element simulations. International Journal for Numerical Methods in Engineering, 60(2):499-526, 2004. ISSN 1097-0207. doi: 10.1002/nme.971.

[23] B. Lé, N. Moës, and G. Legrain. Coupling damage and cohesive zone models with the thick level set approach to fracture. Engineering Fracture Mechanics, 193:214 - 247, 2018. ISSN 0013-7944. doi: https://doi.org/ 10.1016/j.engfracmech.2017.12.036.

[24] J.-J. Marigo. Formulation d'une loi d'endommagement d'un materiau élastique. Comptes Rendus de l'Académie des Sciences. Série II, Jan. 1981

[25] C. Miehe, F. Welschinger, and M. Hofacker. Thermodynamically consistent phase-field models of fracture: Variational principles and multi-field FE implementations. International Journal for Numerical Methods in Engineering, 83(10):1273-1311, 2010.

[26] K. Moreau, N. Moës, N. Chevaugeon, and A. Salzman. Concurrent development of local and non-local damage with the thick level set approach: Implementation aspects and application to quasi-brittle failure. Computer Methods in Applied Mechanics and Engineering, 327:306 - 326, 2017. ISSN 0045-7825. doi: https://doi.org/10.1016/j.cma.2017.08.045. Advances in Computational Mechanics and Scientific Computation-the Cutting Edge.

[27] N. Moës, C. Stolz, P.-E. Bernard, and N. Chevaugeon. A level set based model for damage growth: The thick level set approach. International Journal for Numerical Methods in Engineering, 86(3):358-380, 2011. ISSN 1097-0207. doi: 10.1002/nme.3069.

[28] G. D. Nguyen. A damage model with evolving nonlocal interactions International Journal of Solids and Structures, 48(10):1544-1559, May 2011. ISSN 0020-7683. doi: 10.1016/j.ijsolstr.2011.02.002.

[29] R. H. J. Peerlings, M. G. D. Geers, R. de Borst, and W. A. M. Brekelmans. A critical comparison of nonlocal and gradient-enhanced softening continua. International Journal of Solids and Structures, 38(44):7723-7746, Nov. 2001. ISSN 0020-7683. doi: 10.1016/S0020-7683(01)00087-7.

[30] G. Pijaudier-Cabot and F. Dufour. Non local damage model. European Journal of Environmental and Civil Engineering, 14(6-7):729-749, June 2010. ISSN 1964-8189. doi: 10.1080/19648189.2010.9693260.

[31] G. Pijaudier-Cabot and Z. P. Bažant. Nonlocal Damage Theory. Journal of Engineering Mechanics, 113(10):1512-1533, Oct. 1987. doi: 10.1061/ (ASCE)0733-9399(1987)113:10(1512).

[32] T. Pohl, E. Ramm, and M. Bischoff. Adaptive path following schemes for problems with softening. Finite Elements in Analysis and Design, 86 12-22, Sept. 2014. ISSN 0168-874X. doi: 10.1016/j.finel.2014.02.005.

[33] G. Rastiello, C. Giry, F. Gatuingt, and R. Desmorat. From diffuse damage to strain localization from an Eikonal Non-Local (ENL) Continuum Damage model with evolving internal length. Computer Methods in Applied Mechanics and Engineering, 331:650-674, Apr. 2018. ISSN 0045-7825. doi: 10.1016/j.cma.2017.12.006.

[34] G. Rastiello, F. Riccardi, and B. Richard. Discontinuity-scale pathfollowing methods for the embedded discontinuity finite element modeling of failure in solids. Computer Methods in Applied Mechanics and Engineering, 349:431-457, June 2019. ISSN 0045-7825. doi: 10.1016/j.cma.2019.02.030.

[35] E. Riks. An incremental approach to the solution of snapping and buckling problems. International Journal of Solids and Structures, 15(7):529551, Jan. 1979. ISSN 0020-7683. doi: 10.1016/0020-7683(79)90081-7.

[36] A. Simone, H. Askes, and L. J. Sluys. Incorrect initiation and propagation of failure in non-local and gradient-enhanced media. International journal of solids and structures, 41(2):351-363, 2004.

[37] C. V. Verhoosel and R. de Borst. A phase-field model for cohesive fracture. International Journal for Numerical Methods in Engineering, 96(1): 43-62, 2013. 